\title{
Variation in electron emission time in weakly nonlinear laser wakefield acceleration
}

\author{
K. Huang $\odot,{ }^{1, *}$ H. Kotaki $\odot,{ }^{1}$ M. Mori, ${ }^{1}$ T. Esirkepov ${ }^{1}$ J. K. Koga,${ }^{1}$ Y. Hayashi,${ }^{1}$ N. Nakanii, ${ }^{1}$ \\ S. V. Bulanov, ${ }^{1,2}$ and M. Kando ${ }^{1}$ \\ ${ }^{1}$ Kansai Photon Science Institute, National Institutes for Quantum and Radiological Science \\ and Technology (QST), 8-1-7 Umemidai, Kizugawa-city, Kyoto 619-0215, Japan \\ ${ }^{2}$ Institute of Physics ASCR, v.v.i. (FZU), ELI BEAMLINES, Za Radnicí 835, \\ Dolní Brežany, 252241, Czech Republic
}

(Received 30 May 2019; published 19 December 2019)

\begin{abstract}
As an advanced accelerator concept, laser wakefield acceleration, with its attractive acceleration gradient, has invoked great interest worldwide. The electron beams from laser wakefield acceleration were assumed to have the advantage of "jitterfree" or relatively small jitter, since the emergence of quasimonoenergetic bunch structures were considered to result from the trapping of electrons into the first bucket of the plasma wave. However, the emission times of the electron bunches from laser wakefield acceleration have not been real time monitored in experiments up to now. To examine this, we introduced the electro-optic spatial decoding method. The relative emission times of the electrons were observed to have variations with different plasma densities when using a laser with moderate intensity. The study shows that timing issues should not be ignored in some occasions of laser wakefield acceleration. The method used in this paper could be a candidate serving as a single-shot nondestructive electron bunch timing monitor for laser wakefield experiment and potential ultrafast pump-probe studies.
\end{abstract}

DOI: 10.1103/PhysRevAccelBeams.22.121301

\section{INTRODUCTION}

Laser wakefield acceleration (LWFA) has been studied intensively due to its inherent ultrahigh acceleration gradient $(\mathrm{GeV} / \mathrm{cm})$ and ultrashort pulse duration (fs) compared with conventional accelerators [1-4]. Quasimonoenergetic electron beams with energies over $\mathrm{GeV}$ [5-10], relative energy spread of $0.4 \%$ [11], peak current over kA [12-14] or repetition rate up to $1 \mathrm{kHz}[15,16]$ have been reported by the interaction of intense femtosecond laser pulses with low density gas targets over $\mathrm{mm} \sim \mathrm{cm}$ distances. This compact acceleration regime is considered promising for the construction of table-top ultrafast x-ray sources [17-22] with greater accessibility and lower cost than state of the art light sources driven by conventional accelerators.

For the pump-probe applications of LWFA, the pump or probe lasers are always split out from the drive lasers responsible for the electron beam generation. Other than the ultrashort temporal structure, "jitterfree" or relatively small jitter compared to conventional accelerators is

\footnotetext{
*huang.kai@qst.go.jp
}

Published by the American Physical Society under the terms of the Creative Commons Attribution 4.0 International license. Further distribution of this work must maintain attribution to the author(s) and the published article's title, journal citation, and DOI. considered to be one of the main advantages of laser plasma particle and radiation sources [23-26]. However, the jitterfree characteristic is based on the assumption that electrons are always injected into a certain bucket, in most cases, the first bucket just behind the drive laser pulse in the so called blow-out or "bubble" regime [27,28]. Since the wakewave is composed of multiple wave buckets, the possibility of electron injection into lateral buckets cannot be ignored. The radiated $\mathrm{x}$-ray pulses could have jitter issues in realistic applications. Up to now, the timing of electron bunches from LWFA has received little attention experimentally.

Electro-optic (EO) sampling techniques have been widely used in accelerator physics [29-41]. When an electron bunch passes by an EO crystal, electric fields in the $\mathrm{THz}$ wavelength range copropagating with the electrons act as an external static DC bias possessing temporal information of the electron bunch. A probe laser pulse passing through the crystal at the same time would experience a phase shift of $\Gamma \propto E_{\mathrm{THz}}$ [39], where $E_{\mathrm{THz}}$ is the signal field possessing the temporal information of the electron bunch. By setting a relative angle between the electron beam path and probe laser pulse, the electron bunch temporal information is translated transversely to the probe laser profile nondestructively in a single shot. This is the so called "electro-optic spatial decoding" technique which has been demonstrated to be capable of determining the fluctuation of the electron bunches at femtosecond levels in conventional accelerator research $[34,37,38]$. 

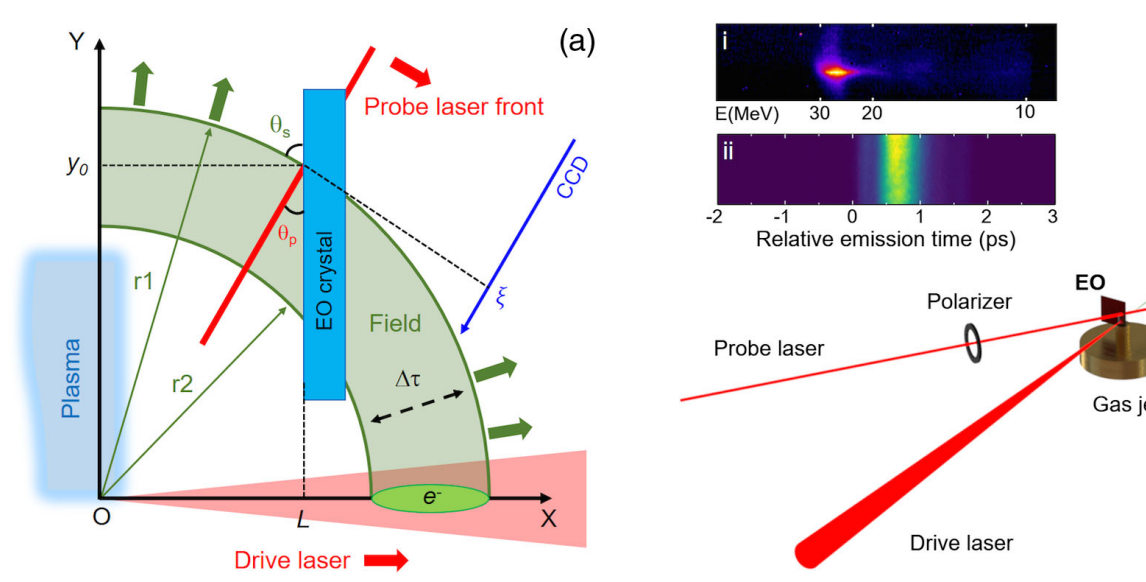

(b)

FIG. 1. Schematic of EO detection. (a) The physics concept of the EO detection when placing the crystal close to the source. Due to the shielding of plasma, the wavefronts of the electric fields have spherical shapes. (b) Experimental setup. The GaP crystal was placed $2.2 \mathrm{~mm}$ from the exit of the plasma in the longitudinal direction. The detection point had a transverse distance $1.5 \mathrm{~mm}$ aside from the drive laser axis. Insets (i) and (ii) are samples of the electron energy spectrum and EO signal recorded simultaneously in a single shot. (iii) is the measured plasma density profiles at two different back pressures with maximum densities of 2.3 (black) and 3.5 (red) $\times 10^{19} \mathrm{~cm}^{-3}$. The blue line denotes the rear edge of the nozzle.

In a previous study [42], we introduced the EO spatial decoding method into a laser wakefield acceleration experiment. By setting an EO crystal at a position very close to the gas target, we observed the field copropagating with the electron bunches had spherical wavefronts due to plasma shielding. Since the electric fields were no longer perpendicular to the electron path, the widely used relationship $c \Delta \tau=\tan \theta_{P} \Delta \xi[36,39]$ was found not applicable, where $\theta_{P}$ is the relative angle between the probe laser and electron path, $\Delta \xi$ and $\Delta \tau$ represent the observed displacement on the CCD and the corresponding time difference. A modified temporal mapping relationship was derived (see Appendix A):

$$
c \Delta \tau=\left(1+\frac{\sin \theta_{S}}{\sin \theta_{P}}\right) \cdot \tan \theta_{P} \Delta \xi
$$

where $\theta_{S}$ represents the incident angle of the signal field onto the crystal surface at the detection point. The physics concept is illustrated as Fig. 1(a).

In this article, we present the observation of the emission time variation of electron beams from LWFA via the EO spatial decoding technique. The relative emission times of the electron beams were discovered to have a dependence on the plasma density for helium (He) gas when using a laser with moderate intensity. The relative "zero" timing was determined by using nitrogen $\left(\mathrm{N}_{2}\right)$ gas. The study suggests that for weakly nonlinear laser wakefield acceleration, attention should be paid to potential jitter issues and injection mechanisms with better controllability should be utilized. This detection method could be introduced as a timing monitor for LWFA experiments and related ultrafast pump-probe studies.

\section{EXPERIMENTAL SETUP}

The experiment was performed on the JLITE-X Ti: Sapphire laser system at the Kansai Photon Science Institute, National Institutes for Quantum and Radiological Science and Technology (QST), Kyoto, Japan. In the experiment, laser pulses with powers of $4 \mathrm{TW}$ and pulse durations of $40 \mathrm{fs}$ (FWHM) were focused using an $F / 20$ off-axis-parabolic (OAP) mirror and incident on a $3 \mathrm{~mm}$ supersonic conical gas jet to generate relativistic electron beams. The focal spot has a $1 / e^{2}$ size of $w_{0}=21 \mu \mathrm{m}$. The resultant laser peak intensity was $\mathrm{I}=7 \times 10^{17} \mathrm{~W} / \mathrm{cm}^{2}$, corresponding to a normalized vector potential $a_{0} \sim 0.57$, where $a_{0}=8.6 \times 10^{-10} \lambda_{0}[\mu \mathrm{m}] \mathrm{I}^{1 / 2}\left[\mathrm{~W} / \mathrm{cm}^{2}\right]$. The electron beams passed through a removable electromagnet coupled with a $2 \mathrm{~mm}$ lead slit and were recorded on a $\mathrm{Gd}_{2} \mathrm{O}_{2} \mathrm{~S}: \mathrm{Tb}$ phosphor screen (Mitsubishi Chemical, DRZ-High). A $100 \mu \mathrm{m} \mathrm{Al}$ filter was placed in front of the DRZ to block visible light. Due to the limit of the set-up, electrons with energy smaller than $10 \mathrm{MeV}$ were not measured. With such a moderate laser intensity, high energy tails in the electron energy spectra were smaller than $50 \mathrm{MeV}$ and a majority of the electrons have energies $<30 \mathrm{MeV}$. The gas back pressure was precisely controlled via a digital controller (General Electric, Druck PACE5000). The neutral gas densities have been measured via a Mach-Zehnder interferometer. The measured density profiles showed that the rear edge of the nozzle resided in the middle of a long down-ramp. The density distributions had similar profiles proportional to the gas back pressures.

To measure the timing information of the electron bunches, a probe laser split from the drive laser was incident through the EO crystal with a relative angle of $\theta_{P}=44^{\circ}$, as illustrated in Fig. 1. In the experiment, the 


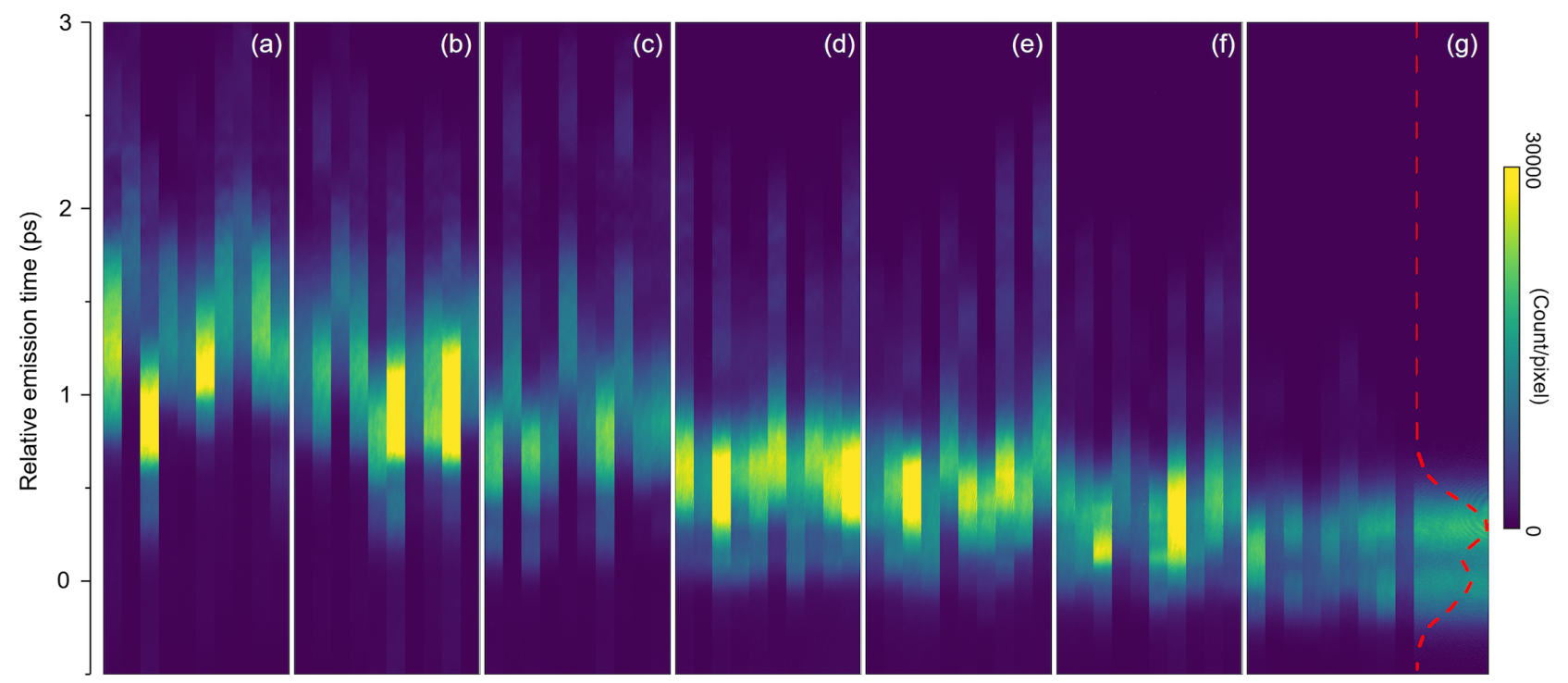

FIG. 2. Single shot EO signals from He. Single-shot EO signals at plasma densities of $\{2.2,2.4,2.7,2.9,3.1,3.5,3.7\} \times 10^{19} \mathrm{~cm}^{-3}$ from He gas are illustrated in (a-g), respectively. 10 consecutive shots of data are listed in each figure with a time range between -0.5 ps and 3 ps. In $(\mathrm{g})$, one of the figures was stretched in width for better visualization of the multibunch structure.

EO signals concurred with the appearance of the electron signals on the phosphor screen, implying that the EO signals were induced by the fields copropagating with the electron beams. Equation (1) has been confirmed by measuring the correspondence between the relative signal displacement and probe delay variation in the experiment at several probe delay settings [42].

Based on the measurement, we got a relationship of $\Delta \tau=1.72 \Delta \xi / c$, corresponding to $6 \mathrm{fs} /$ pixel on the CCD. Note that this is the physical resolution of the EO spatial decoding setup. The real detection resolution is also limited by the relative jitter between the drive laser and the probe laser. Before the EO sampling experiment, by detection on the front edge position of the plasma created by the drive laser, the fluctuation of the relative timing between the drive laser and probe laser was determined to be $21 \mathrm{fs}$. Since the head positions of the plasma represent the ionization fronts, the measured jitters could be caused by the spectrum and phase fluctuations of the laser and vibrations in the optical delivery system. Considering those factors above, in our experimental system, the minimum distinguishable timing difference should be $\lesssim 21$ fs. A detailed discussion of the EO spatial decoding resolution can be found in Appendix D.

\section{OBSERVATION OF THE ELECTRON EMISSION TIME VARIATION}

A list of EO signals of electron bunches from $\mathrm{He}$ for plasma densities from $2.2 \times 10^{19} \mathrm{~cm}^{-3}$ to $3.7 \times 10^{19} \mathrm{~cm}^{-3}$ are illustrated as Fig. 2(a)-2(g) with each figure consisting of 10 consecutive shots. There are three interesting features: (i) Electron beams tended to have emission times earlier at higher plasma densities. (ii) Signals at lower densities have relatively large fluctuations in time, while for higher densities, the center of the signals barely moved. (iii) At higher plasma densities, multibunch structures frequently appeared with timing gaps of $200 \mathrm{fs}-300 \mathrm{fs}$.

A signal line-out in Fig. 2(g) illustrated a two-bunch structure with the first bunch peaked at $8 \mathrm{fs}$ and the second one 264 fs behind. Multibunch structures with similar time gaps have also been reported in Ref. [43-45]. Yet, those works either needed to invasively insert a transition radiation (TR) radiator blocking the beam path or accumulate many shots for one profile. The observations here required much less effort.

The electron timing variation in Fig. 2 was in confliction with the common recognition that laser wakefield accelerated electron beams have the advantage of jitterfree or relatively small jitter. The simultaneously measured electron energy spectrum and EO signal in the insets of Fig. 1(b) also suggested that the emergences of quasimonoenergetic electron bunches are not necessarily due to the so called "blow-out" or "bubble" regime. For many shots, there were small peaks behind the main peaks with time gaps around 1 ps. Those resulted from the reflection of the $\mathrm{THz}$ pulse inside the crystal. Such reflections have also been reported in Ref. [33].

\section{SIMULATION ON THE EMISSION TIME VARIATION}

To understand the electron behaviors in the plasma, we performed two dimensional (2D) particle-in-cell (PIC) simulations using the code REMP [46] (see Appendix C). Figure 3 shows the results of the laser electric fields, plasma density distributions, and the electron $\left(x, p_{x}\right)$ phase spaces when the laser pulses exit the plasma. In the simulation, we 


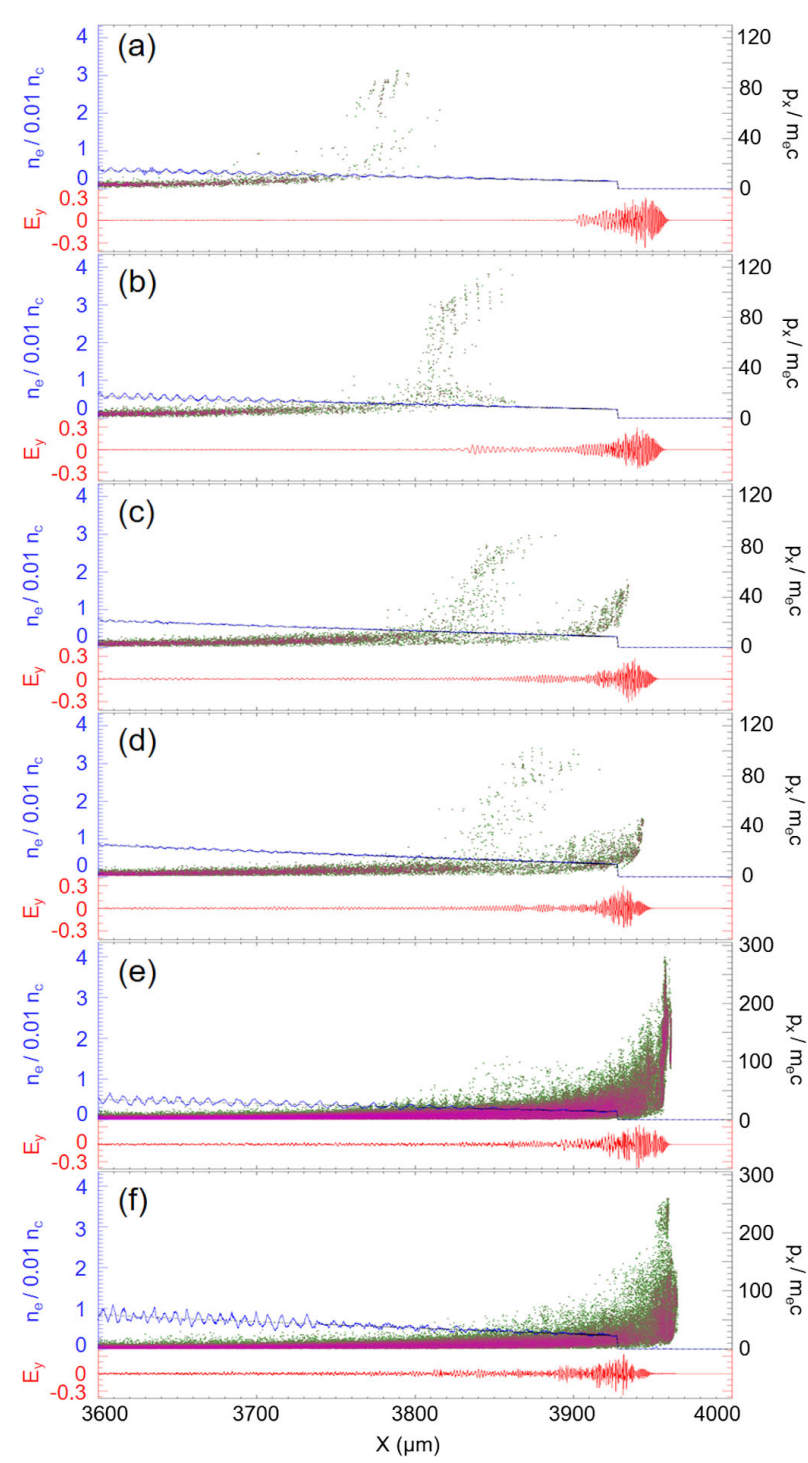

FIG. 3. Particle-in-cell simulations for the pure He case. (a-d) are the simulation results with a lower laser intensity of $a_{0}=0.57$ at plasma densities of $\{2.1,2.4,3.0,3.5\} \times 10^{19} \mathrm{~cm}^{-3}$, respectively. The red plots denote the transverse electric field $\mathrm{E}_{y}$ normalized to $m_{e} \omega_{0} c / e$, where $m_{e}$ is the electron mass and $\omega_{0}$ is the laser frequency. The blue curves denote the electron density profiles in units of $0.01 n_{c}$ which were originally set to be the same as in the experiment, where $n_{c}$ is the critical density for a $800 \mathrm{~nm}$ laser. The electron $\left(x, p_{x}\right)$ phase spaces are plotted out in green (less)-violet (more). Only electrons having $p_{x}>m_{e} c$ are drawn. $(\mathrm{e}, \mathrm{f})$ are the simulation results with a higher laser intensity of $a_{0}=1.13$ at plasma densities of $\{2.1,3.5\} \times 10^{19} \mathrm{~cm}^{-3}$, respectively.

utilized a huge static simulation box with fully ionized plasma. The density profile was set according to the neutral gas density measurement before the experiment. We conducted comparison studies on the same set of plasma densities for two different initial laser intensities with $a_{0}=0.57$ and $a_{0}=1.13$. The simulation results with lower laser intensity at maximum plasma density of $\{2.1,2.4,3.0,3.5\} \times 10^{19} \mathrm{~cm}^{-3}$ are shown as Fig. 3(a)$3(d)$, respectively. We see that the positions of the first group of high energy electrons shift from $\sim 200 \mu \mathrm{m}$ ( $\sim 660 \mathrm{fs}$ ) to 0 relative to the drive laser when increasing the plasma density. Such a phenomenon also appeared in the experimental results in Fig. 2 by checking the front edge positions of the main peaks of the EO signals. In Figs. 3(c) and 3(d), a double-bunch structure with gap of $\sim 100 \mu \mathrm{m}$ ( $\sim 300 \mathrm{fs}$ ) appeared. Such structures were also observed in the experiments as shown in Figs. 2(f) and 2(g). For the higher laser intensity cases at plasma densities of $\{2.1,3.5\} \times 10^{19} \mathrm{~cm}^{-3}$, the electron emission times had no differences, as shown in Figs. 3(e) and 3(f). When using a higher intensity laser, instabilities such as self-modulation and Raman back scattering grow rapidly [47] and wave breaking occurs at a position very close to the laser.

Although a 2D simulation could not be used to repeat what happened in the experiment exactly, the evolution trends of the electron emission time and EO signal patterns with various plasma densities were somehow reproduced. Another point we noted from the simulations was that the EO signals observed in the experiment were contributed by groups of electrons from multiple buckets. This was one of the reasons the EO signals were smeared and electrons from a single bucket were not distinguishable.

\section{DENSITY DEPENDENT EMISSION TIME VARIATION}

Because of the shot by shot timing fluctuations in the EO signals, we performed statistics on 20 signals at each plasma density for the He gas. We choose the center point of the EO signal by doing a single peak Gaussian fit. Such processing should be reasonable considering the field center position of all the energetic electrons rather than a certain electron bunch within a bucket. The mean relative emission time changes from $1.4 \mathrm{ps}$ to $0.16 \mathrm{ps}$ when the plasma density increases from $2.2 \times 10^{19} \mathrm{~cm}^{-3}$ to $3.7 \times 10^{19} \mathrm{~cm}^{-3}$. In the mean time, the standard deviation decreased from 275 fs to 48 fs.

The critical power needed for self-focusing at plasma density $\sim 10^{19} \mathrm{~cm}^{-3}$ is $P_{c}=17 \omega_{0}^{2} / \omega_{p}^{2} \simeq 3 \mathrm{TW}$, where $\omega_{0}$ is the laser frequency and $\omega_{p}=\sqrt{4 \pi n_{e} e^{2} / m_{e}}$ is the plasma frequency [47]. The laser power in the experiment was just above the self-focusing threshold and much lower than the requirement for "blow-out" injection, the electrons could have the possibility to be trapped in the long tail of the wake wave which extends hundreds of micrometers from the drive laser $[48,49]$. We name such a condition as the weakly nonlinear laser wakefield.

We proceed in the discussion of the weakly nonlinear laser wakefield with two assumptions: (i) The first bucket of wake wave contains a similar ratio of laser power for different plasma densities after laser self-focusing. We have the relation: $P=\pi r^{2} I / 2 \propto r^{2} a^{2}$, where $r$ and $a$ denote the 
transverse radius of the bucket and local laser field amplitude at a certain position. Since the self-focusing process is the balance between the laser ponderomotive force and space charge force, we have $k_{p} r=\sqrt{a}$ [47], where $k_{p}$ is the plasma wave number. The dependence of $r$ and $a$ on plasma density $n_{e}$ can be achieved as: $a \propto n_{e}^{1 / 3}$ and $r \propto n_{e}^{-1 / 3}$, respectively. (ii) The long tails of the wake waves work in the linear mode. The maximum wakefield strength is $E_{m}=a^{2} E_{0}$, where $E_{0}=c m \omega_{p} / e$ is the cold nonrelativistic wave breaking field. Although these assumptions might be not suitable to explicitly explain the overall process, they could be helpful for understanding the physics behind what was observed.

For an electron oscillating in a plasma wave $\left(\omega_{p}, k_{p}\right)$, in Lagrangian coordinates, the electron position can be written as $x(t)=x_{0}+\eta(t), x_{0}$ is the position where the electron has maximum velocity, and $\eta(t)=\eta_{m} \cos \left(k_{p} x_{0}-\omega_{p} t\right)$. The electron has an equation of motion: $\partial^{2} x(t) / \partial t^{2}=$ $\partial^{2} \eta(t) / \partial t^{2}=-e E_{w} / m_{e}$, where $E_{w}=E_{m} \cos \left(k_{p} x_{0}-\omega_{p} t\right)$ is the wakefield strength. The maximum displacement is derived as $\eta_{m}=e E_{m} / m_{e} \omega_{p}^{2}=a^{2} c / \omega_{p}$. We conduct the discussion with respect to two basic injection regimes [50,51]: (A) Transverse wave-breaking. The transverse distribution of the laser intensity induces a nonuniform plasma refractive index and results in the so-called "horseshoe" structure, which has been commonly observed in multidimensional PIC simulations and experiments $[48,49]$. A local wave bucket starts to break when the curvature radius of the wake wave is smaller than the electron oscillation amplitude. Although wave breaking occurring in this regime may result in the electron heating rather than injection in certain occasions, we count all the positions where there are possibilities the electron has been trapped into the wakewave. The first place wave-breaking happens has at a distance of $l_{\text {break }} \sim D^{2} / 2 \eta_{m}$ [50] behind the drive laser, where $D=2 r$ is the transverse size of the laser after selffocusing. Thus, the wave breaking time gap relative to the drive laser can be written as:

$$
t=2 r^{2} \omega_{p} / c^{2} a^{2} .
$$

We have a relationship between the injection delay and the plasma density as: $t \propto n^{-5 / 6}$. However, a direct allometric fit to the experiment data (black dashed curve in Fig. 4) shows $t \propto n^{-3.36}$. The fitted index is far from this model. (B) Density down-ramp injection. Electron injections caused by plasma density downramps were frequently observed in experiment [15,52-55]. Considering the plasma has a density inhomogeneity in the longitudinal direction, such a relationship exists at a certain position: $\partial k(x, t) / \partial t=$ $-\partial \omega_{p}(x) / \partial x[51]$. At a certain time $t$ after the laser passes by, the wave number grows as: $k_{p}=k_{0}-\partial_{x} \omega_{p}(x) t$, where $k_{0}=\omega_{p} / v_{g}$ and $v_{g}$ is laser group velocity. With a downramp, the wave number gradually increases and the phase

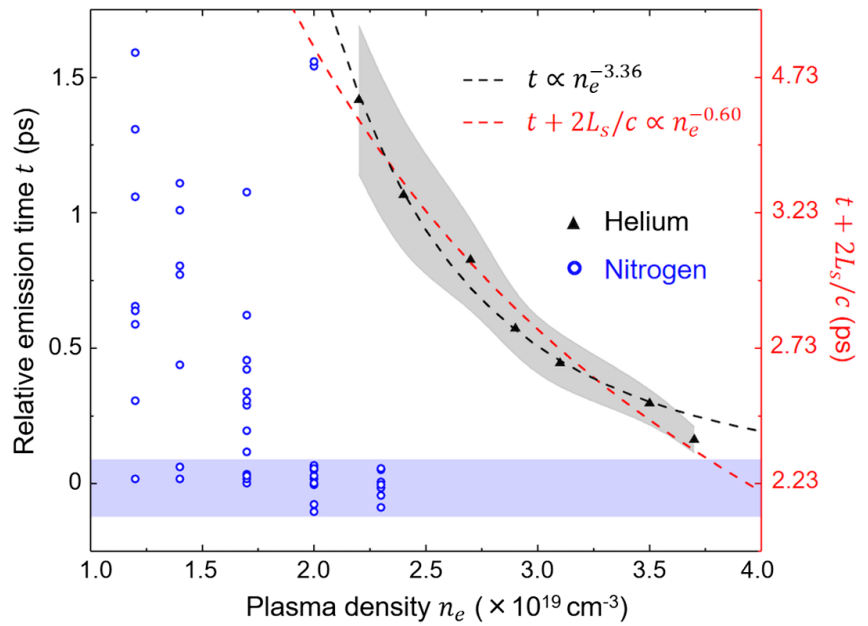

FIG. 4. Discussion of the density dependent emission time variation. The center of the EO signals from He are illustrated by black triangles. The grey shadow in the figure denotes the standard deviations of the relative emission time. The black and red dashed curves are allometric fittings between $\left\{t, n_{e}\right\}$ and $\left\{t+L_{s} / c, n_{e}\right\}$, where $L_{s}$ is the measured plasma scale length of the exit down ramp. The blue circles are the scattering plot of the peak of the signals from $\mathrm{N}_{2}$ for background plasma densities of $\{1.2,1.4,1.7,2.0,2.3\} \times 10^{19} \mathrm{~cm}^{-3}$. The blue zone includes the signals that were taken as samples for relative "zero" timing determination.

velocity of the plasma wave $v_{p}=\omega_{p} / k_{p}$ gradually decreases. When the electron oscillation velocity becomes larger than the plasma wave phase velocity, injection happens. The condition is: $v_{m}=\eta_{m} \omega_{p}=v_{p}$ or $\eta_{m} k_{p}=1$. Thus, for a certain point in the wave, the time when electron trapping happens after the laser passes by is: $t=\left(1 / \eta_{m}-\omega_{p} / v_{g}\right) /\left|\partial_{x} \omega_{p}(x)\right|$. With a density profile of $n_{e}^{\prime}=n_{e} \exp \left(-x / L_{s}\right)$ at the tail of the gas, the plasma frequency has a distribution of $\omega_{p}^{\prime}=\omega_{p} \exp \left(-x / 2 L_{s}\right)$. We have $t=\frac{2 L_{s}}{\omega_{p}}\left(\frac{\omega_{P}}{a^{2} c}-\frac{\omega_{p}}{v_{q}}\right)$. Since $v_{g}$ almost equals c, the relative injection time can be written as:

$$
t=\left(1 / a^{2}-1\right) 2 L_{s} / c .
$$

Rearranging this formula, we get the relationship: $t+2 L_{s} / c=2 L_{s} / c a^{2} \propto 1 / a^{2} \propto n_{e}^{-2 / 3}$. The red dashed curve in Fig. 4 shows a fitted relationship: $t+2 L_{s} / c \propto$ $n_{e}^{-0.60}$. The scale length $L_{s}$ had been measured at various plasma densities to be $484 \mu \mathrm{m}$ with a standard deviation of $55 \mu \mathrm{m}$. The fitted index is somehow quite close to the downramp injection model.

To some extent, this analysis indicates that the trapping of a large portion of the relativistic electrons might happen in the long down-ramp of the plasma for moderate laser intensities. The injection timing of the electrons have a dependence on the steepness of the down-ramp. While increasing the laser intensity or using a high plasma density, both (A) and (B) suggest that wave-breaking may happen at 

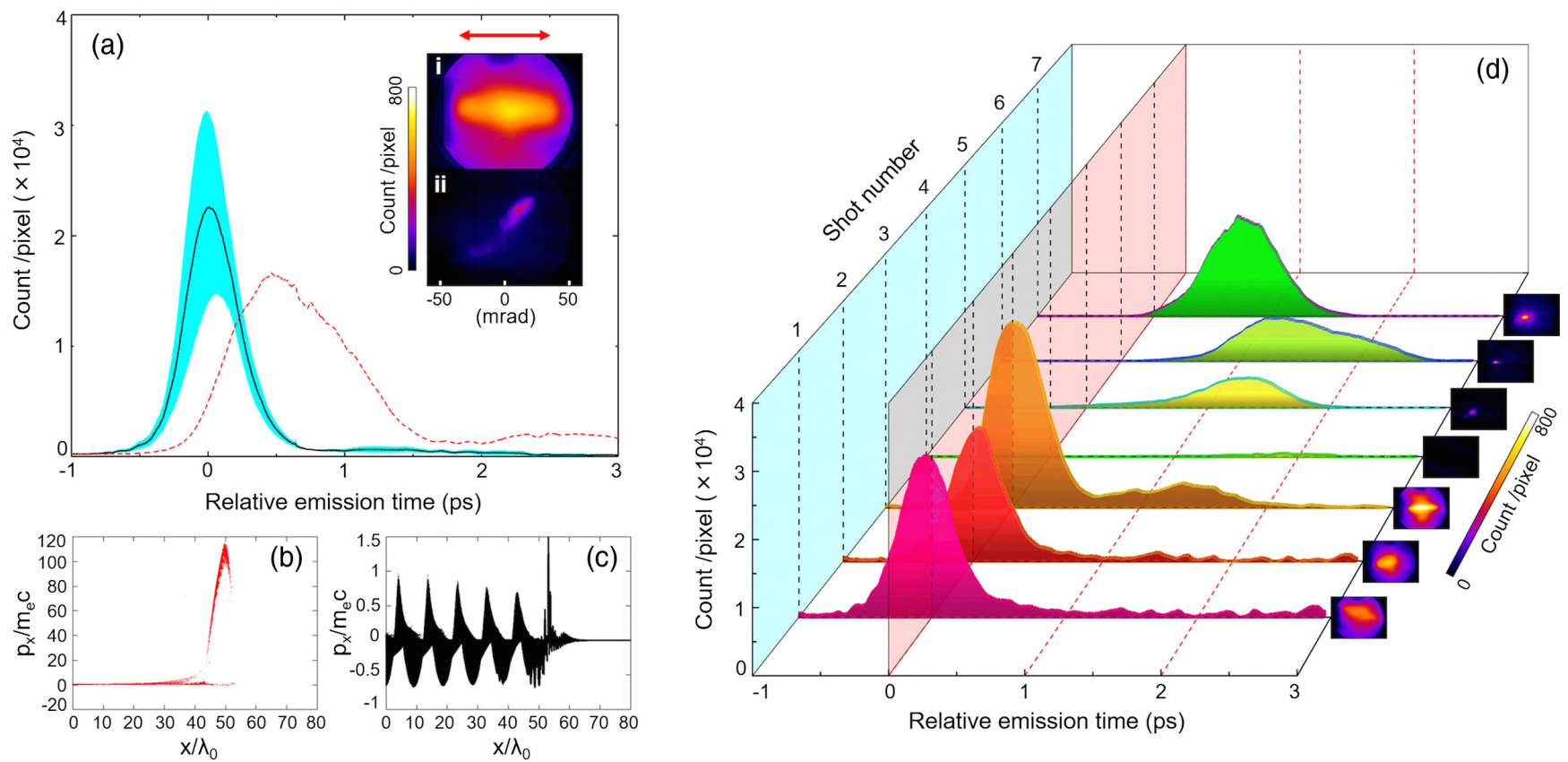

FIG. 5. Relative zero timing determination. (a) The black curve illustrates the mean value from statistics of $28 \mathrm{EO}$ signals from $\mathrm{N}_{2}$ gas for plasma densities from 1.2 to $2.3 \times 10^{19} \mathrm{~cm}^{-3}$. The samples include the signals in the blue zone in Fig. 4 . The shadow in cyan color denotes the standard deviation. The inset (i) in (a) shows the typical electron beam profiles of the shots sampled for zero timing determination. The red double arrow denotes the laser polarization direction. The red dashed curve in (a) is a line out of an EO signal with later timing with its electron profile shown in the inset (ii). (b) and (c) are the ( $\left.x, p_{x}\right)$ phase space plots of the electrons ionized from the K-shell and L-shell of the $\mathrm{N}_{2}$ atoms, respectively, from a PIC simulation at a laser propagation time of 5.3 ps. (d) is a water fall plot of 7 consecutive shots of EO signals at the beginning of the experiment when using pure He gas. The corresponding electron beam profiles are listed to the side.

a position very close to the drive laser, which results in an earlier emission time.

\section{COMPARISON STUDY USING $\mathbf{N}_{2}$}

For comparison, $\mathrm{N}_{2}$ gas was used to confirm the differences of the EO signals when ionization-injection happened. Ionization-injection [56-58] occurs when an inner shell electron is ionized near the peak of the laser and satisfies the condition: $\phi_{f}-\phi_{i}<-m_{e} c^{2} / e$, where $\phi_{i}$ and $\phi_{f}$ denote the field scalar potentials at the position where the electron was born and injected, respectively. The advantage is the inner shell ionized electrons can have higher initial potentials when born and thus easily fulfill the injection requirement.

A scatter plot of the peak positions of the EO signals from $\mathrm{N}_{2}$ can be found in Fig. 4. Different from $\mathrm{He}$, at each plasma density, there were signals appearing near the "zero" timing. At plasma densities $<2 \times 10^{19} \mathrm{~cm}^{-3}$, the EO signals covered a large range of timings. At $2.0 \times 10^{19} \mathrm{~cm}^{-3}$, except for two shots with very late timings, the others were constrained in a region near the zero timing. At $2.3 \times 10^{19} \mathrm{~cm}^{-3}$, no signals were observed with late timings.

On one hand, to work in the ionization-injection regime, the laser should be intense enough to ionize the inner shell electrons. With an initial $a_{0}=0.57$, the ionization probabilities of the K-shell electrons of $\mathrm{N}^{5+}$ ions depended on the laser field strengths after self-focusing, which were influenced by the laser intensity fluctuations and plasma densities. At plasma densities $<2.0 \times 10^{19} \mathrm{~cm}^{-3}$, we had many shots with no electron beams and no EO signals. On the other hand, for an electron to be trapped into buckets close to the drive laser, the plasma wakefield strength should be large enough for the electrons to gain energy quickly when drifting backward. Even though the K-shell electrons were born, the electrons could be trapped into lateral wave buckets, if the wakefield were not strong enough at lower plasma densities. The minimum EO signal standard deviation recorded in the experiment was $30 \mathrm{fs}$ from $\mathrm{N}_{2}$ at $2.3 \times 10^{19} \mathrm{~cm}^{-3}$, which is reasonable including the relative jitter between probe and drive laser ( $\sim 21 \mathrm{fs})$. Based on the measurements, we take the shots in the blue zone of Fig. 4 as samples for the relative "zero" timing determination.

\section{RELATIVE “ZERO” TIMING DETERMINATION}

The black curve in Fig. 5(a) is the mean value from statistics of 28 EO signals in the blue zone of Fig. 4. The peak of the signals were concentrated around the same 
timing point. A 2D simulation using the code EPOCH [59] has been performed to confirm the injection regime (see Appendix C). In the simulation, the initial laser vector potential was $a_{0}=0.57$ and the background plasma density was $2.0 \times 10^{19} \mathrm{~cm}^{-3}$. The $\left(x, p_{x}\right)$ phase space plots in Figs. 5(b) and 5(c) demonstrated that, the high energy electrons are generated from the ionization of the K-shell electrons of $\mathrm{N}_{2}$ ions. The position of the electron beam just resides in the first period of the wake wave. The inset (i) in Fig. 5(a) is a typical electron beam profile of the shots in the blue zone of Fig. 4. The electron beam had an elliptical shape with the long axis along the laser polarization direction, indicating strong interactions between electrons and the laser pulse. For comparison, a line-out of an EO signal with late timing was plotted as a red dashed line in Fig. 5(a). The corresponding electron profile (ii) showed a completely different pattern. With the experimental results and the simulation, we set the peak of the black curve in Fig. 5(a) as the relative zero timing in this paper.

A cross check was performed by analyzing the data of the first several shots in the experiment. When pure He is used in the beginning of the experiment, inevitably, there is residual air in the gas line which mostly consists of Nitrogen, Oxygen, and Carbon. These molecules act as dopants to the pure He and may cause ionization injection. 7 consecutive EO signals and the corresponding electron beam profiles for a background plasma density of $2.2 \times$ $10^{19} \mathrm{~cm}^{-3}$ are illustrated in Fig. 5(d). We observed that the first 3 shots of the EO signals had their main peaks around the relative zero timing and the electron beam profiles had broad elliptical shapes. These features were similar to those in Fig. 5(a). In later shots, the EO signal shifted to later timings and the electron beam had smaller divergences and less charge, which implied that the impurities disappeared and the laser interacted with low density pure $\mathrm{He}$.

\section{DISCUSSION}

The signal fields are mainly composed of the Coulomb field $(\mathrm{CF})$ of an electron itself and the transition radiation (TR) when the electron propagates across the plasma vacuum boundary. For a boundary with step-function profile, the TR should be much larger than the $\mathrm{CF}$ of the electron in the far field where $k R^{\prime} \gg 1$ [60], $k=2 \pi / \lambda$ is the wave number of the radiation and $R^{\prime}$ is the distance from the source to the detection point. Yet, in our experiment, neither of the requirements were satisfied: (i) The gas profile had a gentle down-ramp with a total length of $\sim 1 \mathrm{~mm}$. The polarization responsible for the TR is $P(\omega)=$ $\left(\epsilon_{1}(\omega)-\epsilon_{2}(\omega)\right) E_{q}(\omega) / \pi$ [61], which depends on the plasma density difference on both sides of a certain imaginary boundary, where $\epsilon=1-\omega_{p}^{2} / \omega^{2}$ is the plasma permittivity and $E_{q}$ denotes the local CF strength. A very small density difference would largely weaken the energy radiated away. (ii) The source to detection point distance was merely $2.66 \mathrm{~mm}$. For THz field with wavelength close to millimeter scale, the far-field condition was not well satisfied and the TR field was attached to the CF. An analytic calculation of the TR from such a gentle plasma profile is sophisticated. Based on a previous experiment in Ref. [44], a 10 TW laser interaction with $10^{19} \mathrm{~cm}^{-3}$ plasma would cause the emission of $\mathrm{nC}$ electron beams with temperatures of $5 \mathrm{MeV}$. Such beams generated $\mathrm{THz}$ field with strengths of $<5 \times 10^{4} \mathrm{~V} / \mathrm{m}$ at the detection point in our experiment, which was far below the detection threshold of the EO detection system (see Appendix B).

A simple analysis on the field shape has been performed by calculating the static part of the Lienard-Wiechert potential [61] of a single electron considering the shielding effect with a smooth down-ramp:

$$
\boldsymbol{E}=-\frac{e}{4 \pi \varepsilon_{0}} \frac{\boldsymbol{n}-\boldsymbol{\beta}}{\gamma^{2}(1-\boldsymbol{\beta} \cdot \boldsymbol{n})^{3} R^{2}} f[\beta(c t-R)]_{\mathrm{ret}}
$$

where $\epsilon_{0}$ is the vacuum permittivity, "ret" means the field is calculated at a retarded time, $R$ is the retarded source to observer distance, $\boldsymbol{n}=\boldsymbol{R} / R$ and $\gamma=1 / \sqrt{1-\beta^{2}}$. As shown in Fig. 6(a), at time t, the electron is at position A $(\beta c t, 0)$. The electric field at point $\mathrm{P}$ was generated at an earlier time when the electron was at position $\mathrm{B}$ $(\beta(c t-R), 0)$. For simplicity, the shielding effect has been assumed to be along the electron propagation direction with a function $f(\beta(c t-R))=\{\tanh [\beta(c t-R) / \sigma]+1\} / 2$ defining the transmission ratio, in which $\sigma$ denotes the skewness of the ramp. Since the down-ramp covers $1 \mathrm{~mm}$ in the experiment, a proper value of $\sigma$ would be $250 \mu \mathrm{m}$ considering the profile of a hyperbolic tangent function. The CFs of a $10 \mathrm{MeV}$ electron propagating in free space, passing through a plasma ramp with $\sigma=1 \mu \mathrm{m}$ (almost step function) and $\sigma=250 \mu \mathrm{m}$ are plotted in Fig. 6(b),6(c),6(d), respectively, at $t=8.88 \mathrm{ps}$. Such a time was chosen considering a spherical wave propagates from the source to the detection point $(2.2,1.5 \mathrm{~mm})$ with the speed of $c$. In free space, the $\mathrm{CF}$ is compressed perpendicular to the electron path with a symmetric pattern. With a stepfunction boundary, the $\mathrm{CF}$ is completely constrained in a sphere with a radius of $c t$. We call this sphere the lightfront. With a gentle down-ramp, part of the CF leaks outside of the light front. Nevertheless, the peak positions of the field are still almost located along the light-front marked by the black dashed curve in Fig. 6(d).

To verify this, we plot the temporal profile of the CF field at the detection point, as shown in Fig. 6(e). In the experiment, the longest duration of the EO signal is $<800$ fs (FWHM). Considering the Coulomb field has an transverse broadening angle of $2 / \gamma$, electrons with energy $<6 \mathrm{MeV}$ barely contributed to the signals. In the case of $\sigma=250 \mu \mathrm{m}$, the CFs of an electron with energies of 5,50 , and $500 \mathrm{MeV}$ peak at $\sim 120$ fs after the "light front" and have almost no timing differences. The CF strengths 

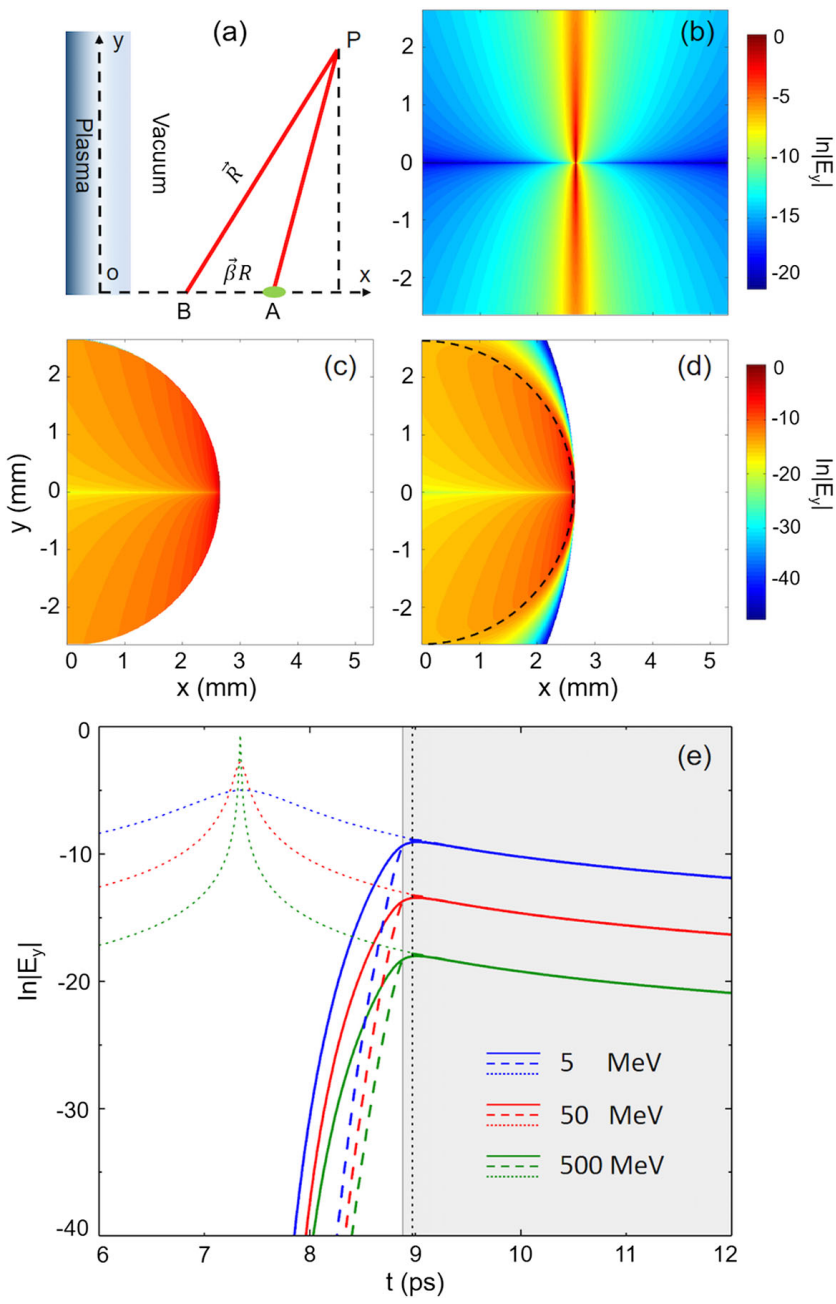

FIG. 6. Discussion on fields structure. (a) is the calculation scheme. (b-d) are the CF spatial distributions of a $10 \mathrm{MeV}$ electron propagating in free space (b), passing through shields with skewness of $1 \mu \mathrm{m}$ (c) and $250 \mu \mathrm{m}$ (d), respectively. In (e), the temporal profiles of the CFs are plotted at the detection point for an electron with an energy of $5 \mathrm{MeV}$ (blue), $50 \mathrm{MeV}$ (red), and $500 \mathrm{MeV}$ (green). The solid and dashed lines denote the field profiles of the electron passing the plasma shield with skewness of $250 \mu \mathrm{m}$ and $100 \mu \mathrm{m}$. The original CF temporal profiles in free space are plotted as colored dotted curves. The grey area shows the region inside the "light front". The dotted black line illustrates the timing of the peak of the CFs for the $\sigma=250 \mu \mathrm{m}$ case.

inside the light-front are weaker for higher energy electrons, because the $\mathrm{CF}$ is concentrated with a factor of $\gamma$ in the perpendicular direction to the electron path and is screened out by the shielding. A portion of the energy screened out is transferred to the TR that is not included in our calculation. The TR field from a step plasma profile would peak at the "light front". However, in the case of a long down-ramp, the electrons produce large part of the TR at the tail of the plasma. The peak of the TR should also shift to a timing later than the light-front and very close to the peak of the CF. Whatever the strength of TR, it should barely have an impact on the timing determination. For comparison, another set of calculations using $\sigma=100 \mu \mathrm{m}$ shows the CFs peak at $100 \mathrm{fs}$ after the light-front. Actually, for various values of skewness and shielding function types, the peaks of the CFs have minor shifts if the centers of the ramp were at the same point. In the experiment, such a point is around the rear edge of the orifice of the conical nozzle.

\section{SUMMARY}

In summary, by introducing the EO spatial decoding method into LWFA, we succeeded monitoring the electron emission time nondestructively in a single shot. A special temporal mapping relationship was used to determine the electron timing when placing the EO crystal close to the target with a large observation angle. Measurements indicated that with a moderate laser intensity, the electron bunches were not necessarily jitterfree. The electron emission time was observed to have a dependence on the plasma density. Relatively controllable injection mechanisms such as ionization injection, down-ramp injection with a steep profile, or colliding pulse injection [62-64] should be applied to get electron bunches with minimum jitter for various applications. This investigation could be helpful for the application of the EO sampling method as a real-time timing monitor for LWFA experiments and related ultrafast pump-probe studies.

\section{ACKNOWLEDGMENTS}

We acknowledge fruitful discussions with Prof. A. Zigler, Prof. P. Bolton, and Dr. A. Pirozhikov, and encouragements from Prof. Hosokai and Dr. Sano. This work was funded by the ImPACT Program of the Council for Science, Technology and Innovation (Cabinet Office, Government of Japan) and the JST-Mirai Program Grant No. JPMJMI17A1, Japan. S. V. B. acknowledges support by the project High Field Initiative (CZ.02.1.01/ 0.0/0.0/15 003/0000449) from the European Regional Development Fund.

\section{APPENDIX A: EO SPATIAL DECODING SETUP}

A pair of nano-particle polarizers (Thorlabs LPVIS100) were inserted before and after the interaction point with polarization direction orthogonal to each other. Due to the relatively low energy and existing energy spread of the electron beam in the experiment, the EO signals were hard to detect at a position far away from the target. Thus, a $50 \mu \mathrm{m}$ thick, [110] cut Gallium Phosphide (GaP) crystal was set $2.2 \mathrm{~mm}$ after the gas jet edge and $1 \mathrm{~mm}$ aside from the laser axis, where damage was not caused by transmitted laser illumination. The detection point has a transverse distance $y=1.5 \mathrm{~mm}$ from the electron beam path. The $[-1,1,0]$ axis of the crystal was aligned along the laser polarization direction. The cross point between the field and EO crystal surface moves with a velocity 
$c<v_{y}=c / \sin \theta_{S}<\infty$. At a certain time, two lines in the signal fields have radius of $r 1$ and $r 2$ with a temporal gap of $\Delta \tau$ between them, as shown in Fig. 1(a). A nonlinear relationship can be achieved by calculating at two time events when the probe front meets with those two signal lines as [42]:

$$
\begin{aligned}
c \Delta \tau= & \Delta \xi \tan \theta_{P}+\frac{L}{\cos \theta_{S}} \\
& -\frac{L}{\cos \theta_{S}} \sqrt{1-\frac{2 \sin \theta_{S} \cos \theta_{S}}{\cos \theta_{P}} \frac{\Delta \xi}{L}+\frac{\cos ^{2} \theta_{S}}{\cos ^{2} \theta_{P}} \frac{\Delta \xi^{2}}{L^{2}}}
\end{aligned}
$$

where $L$ is the longitudinal distance between the plasma exit and the EO crystal. If the investigated process has a very short timescale $(\Delta \xi \ll L)$, the equation above simplifies to equation (1).

\section{APPENDIX B: EVALUATION OF THE EO SIGNAL INTENSITIES}

The extinction ratio of the polarizer pairs had been measured to be $\delta=1.25 \times 10^{-5}$. The phase retardation factor $S_{\text {cross }}=\sin ^{2}(\Gamma / 2)$ is induced by the phase shift, $\Gamma$, which can be calculated as:

$$
\begin{aligned}
\Gamma= & \frac{n_{0}^{3}}{\lambda_{0}} \int_{-\infty}^{+\infty} A(\omega) E_{\mathrm{THz}}(\omega) r_{41}(\omega) e^{i \omega t} \\
& \times \int_{0}^{d} \exp \left[i\left(\frac{\omega}{v_{p h}(\omega)}-\frac{\omega}{v_{g \|}}\right) z\right] \cdot \exp \left[-\frac{\kappa(\omega)}{c} \omega z\right] d z d \omega
\end{aligned}
$$

with the GaP crystal complex refractive index $N(\omega)=$ $n(\omega)+i \kappa(\omega)$, the electro-optic coefficient $r_{41}(\omega)$ and the attenuation factor $A(\omega)$ [41]. $v_{p h}(\omega)=c / n(\omega)$ is the frequency dependent phase velocity of the signal field and $v_{g \|}$ stands for the probe laser group velocity along the signal field propagation direction considering the relative angle between them in the EO crystal. $n_{0}$ is the refractive index of the $\mathrm{GaP}$ crystal for $800 \mathrm{~nm}$ laser. The crystal thickness $d$ is $50 \mu \mathrm{m}$. $E_{\mathrm{THz}}(\omega)$ stands for the frequency domain signal field strength. An observable signal should have $S_{\text {cross }} / \delta>1$. This requires that the $\mathrm{THz}$ field has a strength $>1.2 \times 10^{6} \mathrm{~V} / \mathrm{m}$. A field strength of $5 \times 10^{4} \mathrm{~V} / \mathrm{m}$ results in $S_{\text {cross }} / \delta \sim 0.0015$, which is far below the detection threshold.

For the estimation of the EO signal intensity above, the probe laser elongation in the crystal was not considered. Due to group velocity dispersion (GVD), the pulse duration (FWHM) of the probe laser would be elongated from $\tau_{i}$ to $\tau_{f}=\tau_{0} \sqrt{1+\left(d / L_{\text {char }}\right)^{2}}$ after passing through the crystal, where the characteristic length $L_{\text {char }}=\tau_{i}^{2} /(4 \ln 2$. $\left.d\left(v_{g}^{-1}\right) / d \omega\right)$ [39] depends on the original pulse duration $\tau_{i}$ and refractive index $n_{800}(\omega)$. For a $800 \mathrm{~nm}$ laser pulse with $\tau_{i}=40 \mathrm{fs}$, the characteristic length of a GaP crystal is $L_{\text {char }}=42 \mu \mathrm{m}$. The probe would be elongated to $\tau_{f}=62 \mathrm{fs}$ (FWHM) after propagating through the crystal a with thickness of $d=50 \mu \mathrm{m}$. Such a slight elongation causes little error for the evaluation of the EO signal intensities [41] in this section. Also, since the timing determination process is only concerned with the crosspoint between the peak of the probe laser with the peak of the Coulomb field, as long as the initial pulse duration of the probe laser maintains the same duration, the probe elongation barely has an impact on the resolution of the electron emission time in this paper.

\section{APPENDIX C: PARTICLE-IN-CELL SIMULATIONS}

In this paper, two sets of simulations were conducted: For $\mathrm{He}$, we performed the 2D simulation using the code REMP. Instead of a moving window, we used a huge simulation box with a size of $5500 \lambda_{0} \times 256 \lambda_{0}$ and resolutions of $\Delta x=\lambda_{0} / 16$ and $\Delta y=\lambda_{0} / 8$, where $\lambda_{0}=$ $800 \mathrm{~nm}$ is the laser wavelength. The total macroparticle number is $2.2 \times 10^{9}$ with 12 quasielectrons and 6 quasiions per cell at maximum density. Since the physics process is over picosecond time scales, the ions were set to be mobile; For $\mathrm{N}_{2}$, we performed the $2 \mathrm{D}$ simulation using the code ЕPOCH. A moving window has been employed with size of $80 \lambda_{0} \times 80 \lambda_{0}$ and resolutions of $\Delta x=\lambda_{0} / 32$ and $\Delta y=\lambda_{0} / 16$. We assumed the L-shell electrons had been fully ionized in the front of the laser which is far from the peak and acted as background plasma. The $\mathrm{N}^{5+}$ ions were set to be immobile. There were 10 and 4 macroparticles per cell for the L-shell and K-shell ionized electrons, respectively. Such a simple setup was used to just demonstrate that there was great possibility for the ionized K-shell electrons to be trapped into the first bucket of the wakefield.

\section{APPENDIX D: LIMITATIONS OF MULTIBUNCH STRUCTURE OBSERVATION}

To distinguish the multibunch structure, two factors need to be considered: one is the merging of the Coulomb fields of two electron bunches, the other is the smearing of the EO signal inside the crystal.

\section{Merging of the Coulomb fields of two bunches}

Since the Coulomb field has a transverse opening angle dependent on the electron energy, the broadened fields will merge together and are difficult to be distinguished. The electric field can be estimated using the convolution of $E_{\mathrm{THz}}=\left(E_{r} * Q\right)(r, t)$ [39], where $E_{r}(t)=\frac{e}{4 \pi \varepsilon_{0}} \frac{\gamma r}{\left(r^{2}+\gamma^{2} v^{2} t^{2}\right)^{3 / 2}}$ is the radial electric field of a single electron at distance $r=1.5 \mathrm{~mm}, Q(t)$ is the electron beam temporal distribution and $v$ is the electron velocity. For simplicity, we set the original charge profile of the two bunches as $Q(t)=\frac{1}{\sqrt{2 \pi \sigma_{1}^{2}}} \exp \left(-(t-T c 1)^{2} / 2 \sigma_{1}^{2}\right)+\frac{1}{\sqrt{2 \pi \sigma_{2}^{2}}} \exp \left(-(t-T c 2)^{2} /\right.$ $2 \sigma_{2}^{2}$ ), where $T c 1$ and $T c 2$ are the peak timings of the two 
bunches, $\sigma_{1}$ and $\sigma_{2}$ are the RMS bunch durations, respectively. We introduce a timing gap of 200 fs, i.e., $T c 1=$ -100 fs and $T c 2=+100$ fs. Both bunches are assumed to have 20 fs duration. As shown in Fig. 7(a), the two peaks of the Coulomb fields of $15 \mathrm{MeV}$ electron bunches are difficult to distinguish, while the fields from $500 \mathrm{MeV}$ electrons maintain the original shapes. In our experiment, a majority of the electrons have energies $<30 \mathrm{MeV}$, merging of the Coulomb field exists. The reason why we choose a bunch duration of $20 \mathrm{fs}$ as an example is that for plasma density $>2 \times 10^{19} \mathrm{~cm}^{-3}$, the size of a single plasma bucket is smaller than $7.38 \mu \mathrm{m}(1 \mu \mathrm{m} \sim 3.3 \mathrm{fs})$.

\section{Smearing of the Coulomb fields coding process inside the $\mathrm{EO}$ crystal}

The smearing process is illustrated in Fig. 7(b). The Coulomb field and probe laser have propagation angles of $\theta_{S}^{\prime}$ and $\theta_{P}^{\prime}$ inside the crystal relative to the crystal surface. Correspondingly, the incident angles of the Coulomb field and the probe laser are $\theta_{S}$ and $\theta_{P}$, respectively. The coding process begins when the cross point between the probe laser and Coulomb field front enters the crystal (point A, at time $t_{A}$ ), and ends when the cross point goes out of the crystal (point $\mathrm{B}$, at time $t_{B}$ ). The total coding process takes time $t$. The effective propagation distances of Coulomb field and the probe laser inside the crystal are $v_{S} t$ and $v_{P} t$, where $v_{S}$ and $v_{P}$ are the phase velocity of the Coulomb field and the group velocity of probe laser inside the crystal, respectively. The crystal has a thickness of $d=50 \mu \mathrm{m}$. The smearing region is plotted in green color between point $A^{\prime}$ to $B$. From the geometry shown in Fig. 7(b), we have relationships of: (i) $v_{P} t=d / \cos \theta_{P}^{\prime}-X_{s} \cdot \tan \theta_{P}^{\prime}$ and (ii) $v_{S} t=v_{P} t \cdot \cos \left(\theta_{P}^{\prime}+\theta_{S}^{\prime}\right)+X_{S} \cdot \sin \left(\theta_{P}^{\prime}+\theta_{S}^{\prime}\right)$. Defining the relative angle between the probe and Coulomb field inside the EO crystal as $\alpha=\theta_{P}^{\prime}+\theta_{S}^{\prime}$, we achieve the transverse smearing length on the probe wavefront (inside the crystal):

$$
X_{s}=d /\left(\frac{v_{P} \sin \alpha \cos \theta_{P}^{\prime}}{v_{S}-v_{P} \cos \alpha}+\sin \theta_{P}^{\prime}\right) .
$$

The smearing region after the probe going out of the crystal can be calculated considering the refraction of the probe laser $n_{P}: \Delta \xi=X_{s} \frac{\cos \theta_{P}}{\cos \theta_{P}^{\prime}}=d \cdot \cos \theta_{P} /$ $\left(\frac{v_{P} \sin \alpha \cos ^{2} \theta_{P}^{\prime}}{v_{S}-v_{P} \cos \alpha}+\sin \theta_{P}^{\prime} \cos \theta_{P}^{\prime}\right)$. Using the temporal mapping relationship elaborated as Eq. (1), we achieve the smearing time:

$$
\Delta \tau=\frac{\sin \theta_{P}+\sin \theta_{S}}{\frac{v_{P} \sin \alpha \cos ^{2} \theta_{P}^{\prime}}{v_{S}-v_{P} \cos \alpha}+\sin \theta_{P}^{\prime} \cos \theta_{P}^{\prime}} \cdot d / c .
$$

For the investigation and optimization of the EO spatial decoding method in the future, we introduce a smearing
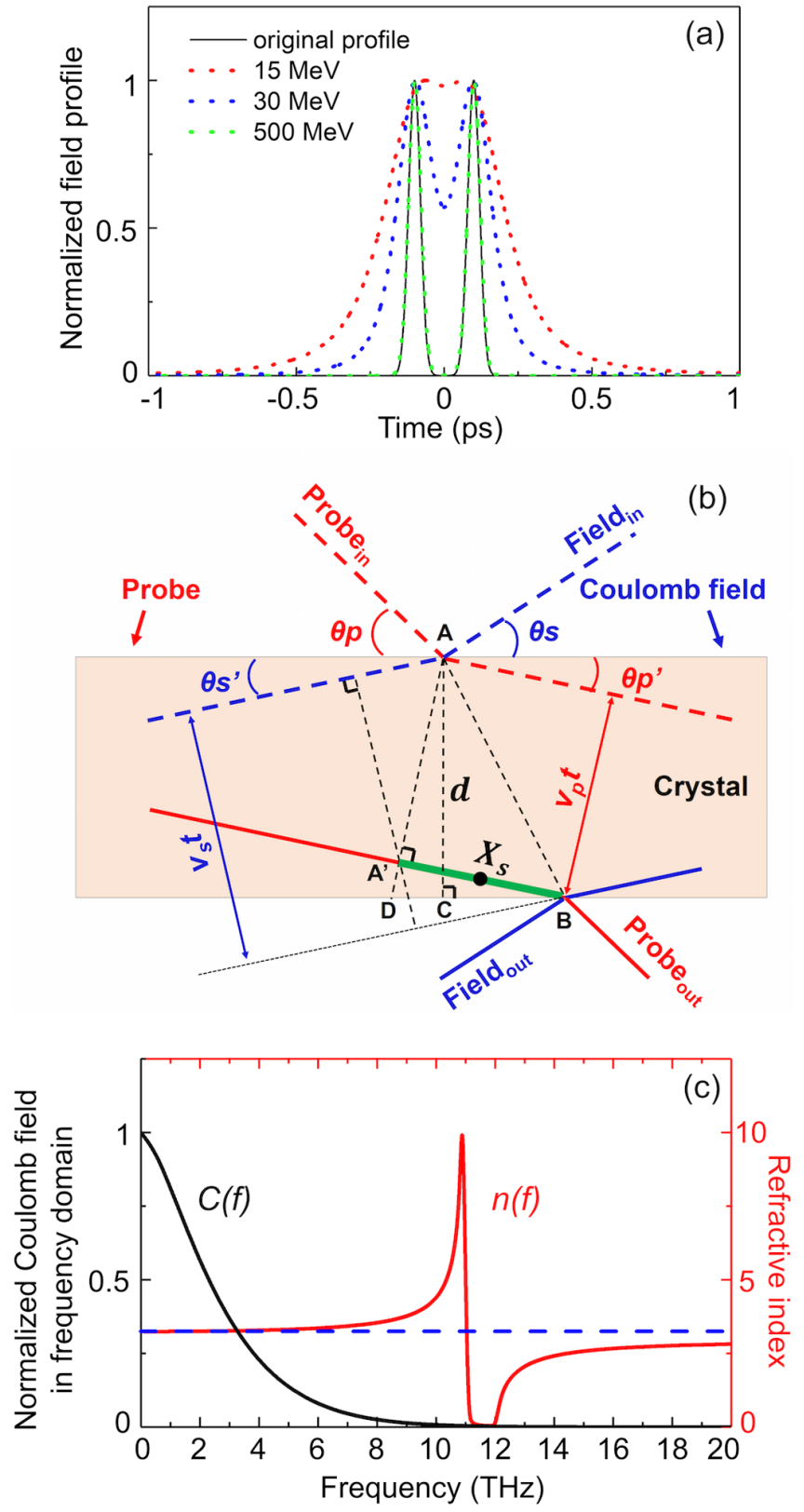

FIG. 7. Discussion on the limitation of EO spatial decoding. (a) Coulomb fields of a double bunch structure. The black curve is the original charge shape. Red, blue and green dotted lines denote the fields of $15 \mathrm{MeV}, 30 \mathrm{MeV}$, and $500 \mathrm{MeV}$ electrons, respectively. (b) Smearing process inside the EO crystal. "A" and " $\mathrm{B}$ " denote the beginning $\left(t_{A}\right)$ and end $\left(t_{B}\right)$ of the EO coding process. The blue dashed and solid lines show the peaks of the Coulomb fields at time $t_{A}$ and $t_{B}$, while the red lines illustrate those of the probe laser. The crystal has a thickness of $d$. The green line denotes the smearing part of the signal with a length of $X_{s}$. The black dot at the center of the smearing part denotes the peak of the EO signal. (c) The frequency domain Coulomb field of a $30 \mathrm{MeV}, 20$ fs electron bunch (black curve) and the refractive index of $\mathrm{GaP}$ crystal in the $\mathrm{THz}$ range (red curve). The blue dashed line denotes an average refractive index of $\bar{n}_{S}=3.267$ for the estimation of the propagation angle and phase velocity of the Coulomb field inside the crystal. 
factor $S$ with the relationship of $c \Delta \tau=S \cdot d$. The smearing factor $S$ can be understood as the ratio of the smearing length observed by the CCD $\Delta \xi$ to the thickness of the crystal $d$. $S$ can be calculated as:

$$
S=\frac{\sin \theta_{P}+\sin \theta_{S}}{\frac{v_{P} \sin \alpha \cos ^{2} \theta_{P}^{\prime}}{v_{S}-v_{P} \cos \alpha}+\sin \theta_{P}^{\prime} \cos \theta_{P}^{\prime}} .
$$

The group velocity of $800 \mathrm{~nm}$ light in $\mathrm{GaP}$ is $v_{P}=$ $0.280 c$. The propagation angles inside the EO crystal are determined by the refractive indexes. The refractive index of the probe laser in GaP crystal is $n_{P}=3.183$, which is calculated by $n_{P}(\lambda)=\sqrt{2.680+6.40 \lambda^{2} /\left(\lambda^{2}-0.0903279\right)}$, where $\lambda$ is the laser wavelength in $\mu \mathrm{m}$. The Coulomb field in the $\mathrm{THz}$ range is polychromatic. In our experiment, a majority of the electrons reside in energy range $<30 \mathrm{MeV}$, for estimation, the frequency domain Coulomb field of an electron bunch with duration of $20 \mathrm{fs}$ and energy of $30 \mathrm{MeV}$ is calculated and illustrated as the black curve $C(f)$ in Fig. $7(\mathrm{c})$, where $C(f)=\int E_{\mathrm{THz}}(t) e^{i 2 \pi f t} d t$. Due to the broadening effect, the majority of the Fourier components have frequencies smaller than $6 \mathrm{THz}$. The average refractive index is estimated via the refractive index curve $n(f)$ [39] in Fig. 7(c) (red curve): $\bar{n}_{S}=\Sigma[C(f) n(f)] / \Sigma C(f)=3.267$. Thus, the phase velocity of the Coulomb field inside the crystal is estimated as $v_{S}=c / \bar{n}_{S}=0.306 c$. From the temporal mapping relationship measured in the experiment $c \Delta \tau=1.72 \Delta \xi$, the incident angle of the Coulomb field can be deduced via Eq. (1) to be $\theta_{S}=32.861^{\circ}$, while the probe laser has an incident angle of $\theta_{P}=44^{\circ}$. The angles inside the crystal are: $\theta_{S}^{\prime}=\arcsin \left(\sin \theta_{S} / \bar{n}_{S}\right)=9.560^{\circ}, \quad \theta_{P}^{\prime}=$ $\arcsin \left(\sin \theta_{P} / n_{P}\right)=12.606^{\circ}$, and $\alpha=\theta_{P}^{\prime}+\theta_{S}^{\prime}=22.166^{\circ}$. Using Eq. (D3), the smearing factor $S=0.523$, corresponding to a smearing time of $\Delta \tau \sim 87$ fs for a crystal thickness of $d=50 \mu \mathrm{m}$.

The analysis above indicates that, for the geometry of the EO setup in our experiment, the minimum observable gap between two separate bunches is $>87 \mathrm{fs}$, even for the case where electrons have very high energy and the broadening effect of the Coulomb field is negligible. For electrons with energy $<30 \mathrm{MeV}$, since both the smearing of the EO coding process and the merging of Coulomb fields exist, it is difficult to distinguish two electron bunches with a gap $<200$ fs. These are two reasons why all the observed twobunch structures have a timing gap $>200$ fs in the experiment. Equation (D3) suggests that to achieve a better resolution for the observation of the multibunch structure, a thinner EO crystal and smaller relative angle between the probe laser and the Coulomb field should be utilized. Also, searching for new materials with dispersion properties allowing for smaller difference between the group velocity of the probe and the phase velocity of the Coulomb field is of great significance.
We want to point out that, although smearing happens, it does not affect the determination of the relative emission time. The peak of the observed EO signal should reside at the black dot in Fig. 7(b), which is the center of the smearing part. For an electron with different emission time, the field has almost the same width of the smearing region. Because a majority of the Fourier components of the Coulomb field have similar refractive indexes, they almost have the same propagation angle and phase velocity inside the EO crystal.

[1] T. Tajima and J. M. Dawson, Laser Electron Accelerator, Phys. Rev. Lett. 43, 267 (1979).

[2] J. Faure, Y. Glinec, A. Pukhov, S. Kiselev, S. Gordienko, E. Lefebvre, J.-P. Rousseau, F. Burgy, and V. Malka, A laserplasma accelerator producing monoenergetic electron beams, Nature (London) 431, 541 (2004).

[3] S. P. D. Mangles, C. D. Murphy, Z. Najmudin, A. G. R. Thomas, J. L. Collier, A. E. Dangor, E. J. Divall, P. S. Foster, J. G. Gallacher, C. J. Hooker et al., Monoenergetic beams of relativistic electrons from intense laser-plasma interactions, Nature (London) 431, 535 (2004).

[4] C. G. R. Geddes, C. s. Toth, J. Van Tilborg, E. Esarey, C. B. Schroeder, D. Bruhwiler, C. Nieter, J. Cary, and W. P. Leemans, High-quality electron beams from a laser wakefield accelerator using plasma-channel guiding, Nature (London) 431, 538 (2004).

[5] W. P. Leemans, B. Nagler, A. J. Gonsalves, C. s. Toth, K. Nakamura, C. G. R. Geddes, E. S. C. B. Esarey, C. B. Schroeder, and S. M. Hooker, Gev electron beams from a centimetre-scale accelerator, Nat. Phys. 2, 696 (2006).

[6] W. P. Leemans, A. J. Gonsalves, H-S Mao, K. Nakamura, C. Benedetti, C. B. Schroeder, C. s. Tóth, J. Daniels, D. E. Mittelberger, S.S. Bulanov et al., Multi-Gev Electron Beams from Capillary-Discharge-Guided Subpetawatt Laser Pulses in the Self-Trapping Regime, Phys. Rev. Lett. 113, 245002 (2014).

[7] A. J. Gonsalves, K. Nakamura, J. Daniels, C. Benedetti, C. Pieronek, T. C. H. de Raadt, S. Steinke, J. H. Bin, S. S. Bulanov, J. van Tilborg et al., Petawatt Laser Guiding and Electron Beam Acceleration to $8 \mathrm{Gev}$ in a Laser-Heated Capillary Discharge Waveguide, Phys. Rev. Lett. 122, 084801 (2019).

[8] X. Wang, R. Zgadzaj, N. Fazel, Z. Li, S. A. Yi, X. Zhang, W. Henderson, Y.-Y. Chang, R. Korzekwa, H.-E. Tsai et al., Quasi-monoenergetic laser-plasma acceleration of electrons to $2 \mathrm{Gev}$, Nat. Commun. 4, 1988 (2013).

[9] H. T. Kim, K. H. Pae, H. J. Cha, I. J. Kim, T. J. Yu, J. H. Sung, S. K. Lee, T. M. Jeong, and J. Lee, Enhancement of Electron Energy to the Multi-Gev Regime by a Dual-Stage Laser-Wakefield Accelerator Pumped by Petawatt Laser Pulses, Phys. Rev. Lett. 111, 165002 (2013).

[10] M. Mirzaie, S. Li, M. Zeng, N. A. M. Hafz, M. Chen, G. Y. Li, Q. J. Zhu, H. Liao, T. Sokollik, F. Liu et al., Demonstration of self-truncated ionization injection for $\mathrm{Gev}$ electron beams, Sci. Rep. 5, 14659 (2015). 
[11] W. T. Wang, W. T. Li, J. S. Liu, Z. J. Zhang, R. Qi, C. H. Yu, J. Q. Liu, M. Fang, Z. Y. Qin, C. Wang et al., HighBrightness High-Energy Electron Beams from a Laser Wakefield Accelerator via Energy Chirp Control, Phys. Rev. Lett. 117, 124801 (2016).

[12] O. Lundh, J. Lim, C. Rechatin, L. Ammoura, A. BenIsmail, X. Davoine, G. Gallot, J.-P. Goddet, E. Lefebvre, V. Malka et al., Few femtosecond, few kiloampere electron bunch produced by a laser-plasma accelerator, Nat. Phys. 7, 219 (2011).

[13] J. P. Couperus, R. Pausch, A. Köhler, O. Zarini, J. M. Krämer, M. Garten, A. Huebl, R. Gebhardt, U. Helbig, S. Bock et al., Demonstration of a beam loaded nanocoulomb-class laser wakefield accelerator, Nat. Commun. 8, 487 (2017).

[14] Y. F. Li, D.Z. Li, K. Huang, M.Z. Tao, M. H. Li, J. R. Zhao, Y. Ma, X. Guo, J. G. Wang, M. Chen et al., Generation of 20 ka electron beam from a laser wakefield accelerator, Phys. Plasmas 24, 023108 (2017).

[15] Z. H. He, B. Hou, J. A. Nees, J. H. Easter, J. Faure, K. Krushelnick, and A. G. R. Thomas, High repetition-rate wakefield electron source generated by few-millijoule, 30 fs laser pulses on a density downramp, New J. Phys. 15, 053016 (2013).

[16] D. Guénot, D. Gustas, A. Vernier, B. Beaurepaire, F. Böhle, M. Bocoum, M. Lozano, A. Jullien, R. Lopez-Martens, A. Lifschitz et al., Relativistic electron beams driven by $\mathrm{KHz}$ single-cycle light pulses, Nat. Photonics 11, 293 (2017).

[17] K. Nakajima, Compact x-ray sources: Towards a table-top free-electron laser, Nat. Phys. 4, 92 (2008).

[18] M. Fuchs, R. Weingartner, A. Popp, Z. Major, S. Becker, J. Osterhoff, I. Cortrie, B. Zeitler, R. Hörlein, G. D. Tsakiris et al., Laser-driven soft-x-ray undulator source, Nat. Phys. 5, 826 (2009).

[19] A. Rousse, K. Ta Phuoc, R. Shah, A. Pukhov, E. Lefebvre, V. Malka, S. Kiselev, F. Burgy, J.-P. Rousseau, D. Umstadter et al., Production of a Kev X-ray Beam from Synchrotron Radiation in Relativistic Laser-Plasma Interaction, Phys. Rev. Lett. 93, 135005 (2004).

[20] K. Huang, Y. F. Li, D. Z. Li, L. M. Chen, M. Z. Tao, Y. Ma, J. R. Zhao, M. H. Li, M. Chen, M. Mirzaie et al., Resonantly enhanced betatron hard $\mathrm{X}$-rays from ionization injected electrons in a laser plasma accelerator, Sci. Rep. 6, 27633 (2016).

[21] M. Kando, A. S. Pirozhkov, K. Kawase, T. Zh Esirkepov, Y. Fukuda, H. Kiriyama, H. Okada, I. Daito, T. Kameshima, Y. Hayashi et al., Enhancement of Photon Number Reflected by the Relativistic Flying Mirror, Phys. Rev. Lett. 103, 235003 (2009).

[22] W. Yan, C. Fruhling, G. Golovin, D. Haden, J. Luo, P. Zhang, B. Zhao, J. Zhang, C. Liu, M. Chen et al., Highorder multiphoton thomson scattering, Nat. Photonics 11, 514 (2017).

[23] K. Ta Phuoc, R. Fitour, A. Tafzi, T. Garl, N. Artemiev, R. Shah, F. Albert, D. Boschetto, A. Rousse, D. E. Kim et al., Demonstration of the ultrafast nature of laser produced betatron radiation, Phys. Plasmas 14, 080701 (2007).

[24] F. Albert, A. G. R. Thomas, S. P. D. Mangles, S. Banerjee, S. Corde, A. Flacco, M. Litos, D. Neely, J. Vieira, Z.
Najmudin et al., Laser wakefield accelerator based light sources: potential applications and requirements, Plasma Phys. Controlled Fusion 56, 084015 (2014).

[25] B. Mahieu, N. Jourdain, K. Ta Phuoc, F. Dorchies, J-P. Goddet, A. Lifschitz, P. Renaudin, and L. Lecherbourg, Probing warm dense matter using femtosecond $\mathrm{x}$-ray absorption spectroscopy with a laser-produced betatron source, Nat. Commun. 9, 3276 (2018).

[26] A. Buck, M. Nicolai, K. Schmid, C. M. S. Sears, A. Sävert, J. M. Mikhailova, F. Krausz, M. C. Kaluza, and L. Veisz, Real-time observation of laser-driven electron acceleration, Nat. Phys. 7, 543 (2011).

[27] A. Pukhov and J. Meyer-ter Vehn, Laser wake field acceleration: The highly non-linear broken-wave regime, Appl. Phys. B 74, 355 (2002).

[28] W. Lu, C. Huang, M. Zhou, W. B. Mori, and T. Katsouleas, Nonlinear Theory for Relativistic Plasma Wakefields in the Blowout Regime, Phys. Rev. Lett. 96, 165002 (2006).

[29] X. Yan, A. M. MacLeod, W. A. Gillespie, G. M. H. Knippels, D. Oepts, A. F. G. van der Meer, and W. Seidel, Subpicosecond Electro-optic Measurement of Relativistic Electron Pulses, Phys. Rev. Lett. 85, 3404 (2000).

[30] I. Wilke, Allan M. MacLeod, W. Allan Gillespie, G. Berden, G. M. H. Knippels, and A. F. G. Van Der Meer, Single-Shot Electron-Beam Bunch Length Measurements, Phys. Rev. Lett. 88, 124801 (2002).

[31] G. Berden, S. P. Jamison, A. M. MacLeod, W. A. Gillespie, B. Redlich, and A. F. G. Van der Meer, Electro-optic Technique with Improved Time Resolution for Real-Time, Nondestructive, Single-Shot Measurements of Femtosecond Electron Bunch Profiles, Phys. Rev. Lett. 93, 114802 (2004).

[32] G. Berden, W. Allan Gillespie, S. P. Jamison, E-A. Knabbe, A. M. MacLeod, A. F. G. Van Der Meer, P. J. Phillips, H. Schlarb, B. Schmidt, P. Schmüser et al., Benchmarking of Electro-optic Monitors for Femtosecond Electron Bunches, Phys. Rev. Lett. 99, 164801 (2007).

[33] B. Steffen, V. Arsov, G. Berden, W. Allan Gillespie, S. P. Jamison, Allan M. MacLeod, A. F. G. Van Der Meer, P. J. Phillips, H. Schlarb, B. Schmidt et al., Electro-optic time profile monitors for femtosecond electron bunches at the soft x-ray free-electron laser flash, Phys. Rev. Accel. Beams 12, 032802 (2009).

[34] A. L. Cavalieri, D. M. Fritz, S. H. Lee, P. H. Bucksbaum, D. A. Reis, J. Rudati, D. M. Mills, P. H. Fuoss, G. B. Stephenson, C. C. Kao et al., Clocking Femtosecond X Rays, Phys. Rev. Lett. 94, 114801 (2005).

[35] X. Yang, T. Tsang, T. Rao, J. B. Murphy, Y. Shen, and X. J. Wang, Electron bunch length monitors using spatially encoded electro-optical technique in an orthogonal configuration, Appl. Phys. Lett. 95, 231106 (2009).

[36] W. Wang, Y. Du, L. Yan, Z. Chi, Z. Zhang, J. Hua, W. Huang, C. Tang, and M. Li, Temporal profile monitor based on electro-optic spatial decoding for lowenergy bunches, Phys. Rev. Accel. Beams 20, 112801 (2017).

[37] C. M. Scoby, P. Musumeci, J. T. Moody, and M. S. Gutierrez, Electro-optic sampling at 90 degree interaction geometry for time-of-arrival stamping of ultrafast relativ- 
istic electron diffraction, Phys. Rev. Accel. Beams 13, 022801 (2010).

[38] S. P. Jamison, G. Berden, Allan M. MacLeod, D. A. Jaroszynski, B. Redlich, A. F. G. Van der Meer, and W. Allan Gillespie, Electro-optic techniques for temporal profile characterisation of relativistic coulomb fields and coherent synchrotron radiation, Nucl. Instrum. Methods Phys. Res., Sect. A 557, 305 (2006).

[39] Bernd Richard Steffen, Electro-optic methods for longitudinal bunch diagnostics at FLASH, Ph.D. thesis, Hamburg U., 2007.

[40] D. Sütterlin, D. Erni, V. Schlott, H. Sigg, H. Jäckel, and A. Murk, Single-shot electron bunch length measurements using a spatial electro-optical autocorrelation interferometer, Rev. Sci. Instrum. 81, 104702 (2010).

[41] S. Casalbuoni, H. Schlarb, B. Schmidt, P. Schmüser, B. Steffen, and A. Winter, Numerical studies on the electrooptic detection of femtosecond electron bunches, Phys. Rev. Accel. Beams 11, 072802 (2008).

[42] K. Huang, T. Esirkepov, J. K. Koga, H. Kotaki, M. Mori, Y. Hayashi, N. Nakanii, S. V. Bulanov, and M. Kando, Electro-optic spatial decoding on the spherical-wavefront coulomb fields of plasma electron sources, Sci. Rep. 8, 2938 (2018).

[43] A. D. Debus, M. Bussmann, U. Schramm, R. Sauerbrey, C. D. Murphy, Z. Major, R. Hörlein, L. Veisz, K. Schmid, J. Schreiber et al., Electron Bunch Length Measurements from Laser-Accelerated Electrons Using Single-Shot THz Time-Domain Interferometry, Phys. Rev. Lett. 104, 084802 (2010).

[44] J. Van Tilborg, C. B. Schroeder, C. V. Filip, C. s. Tóth, C. G. R. Geddes, G. Fubiani, Rupert Huber, R. A. Kaindl, E. Esarey, and W. P. Leemans, Temporal Characterization of Femtosecond Laser-Plasma-Accelerated Electron Bunches Using Terahertz Radiation, Phys. Rev. Lett. 96, 014801 (2006).

[45] M. Heigoldt, A. Popp, K. Khrennikov, J. Wenz, S.-W. Chou, S. Karsch, S. I. Bajlekov, S. M. Hooker, and B. Schmidt, Temporal evolution of longitudinal bunch profile in a laser wakefield accelerator, Phys. Rev. Accel. Beams 18, 121302 (2015).

[46] T. Zh. Esirkepov, Exact charge conservation scheme for particle-in-cell simulation with an arbitrary form-factor, Comput. Phys. Commun. 135, 144 (2001).

[47] E. Esarey, C. B. Schroeder, and W. P. Leemans, Physics of laser-driven plasma-based electron accelerators, Rev. Mod. Phys. 81, 1229 (2009).

[48] S Yu Kalmykov, L. M. Gorbunov, P. Mora, and G. Shvets, Injection, trapping, and acceleration of electrons in a threedimensional nonlinear laser wakefield, Phys. Plasmas 13, 113102 (2006).

[49] N. H. Matlis, S. Reed, S. S. Bulanov, V. Chvykov, G. Kalintchenko, T. Matsuoka, P. Rousseau, V. Yanovsky, A. Maksimchuk, S. Kalmykov et al., Snapshots of laser wakefields, Nat. Phys. 2, 749 (2006).

[50] S. V. Bulanov, Francesco Pegoraro, A. M. Pukhov, and A. S. Sakharov, Transverse-Wake Wave Breaking, Phys. Rev. Lett. 78, 4205 (1997).
[51] S. Bulanov, N. Naumova, F. Pegoraro, and J. Sakai, Particle injection into the wave acceleration phase due to nonlinear wake wave breaking, Phys. Rev. E 58, R5257 (1998).

[52] C. G. R. Geddes, K. Nakamura, G. R. Plateau, C. s. Toth, E. Cormier-Michel, E. Esarey, C. B. Schroeder, J. R. Cary, and W. P. Leemans, Plasma-Density-Gradient Injection of Low Absolute-Momentum-Spread Electron Bunches, Phys. Rev. Lett. 100, 215004 (2008).

[53] A. J. Gonsalves, K. Nakamura, C. Lin, D. Panasenko, S. Shiraishi, T. Sokollik, C. Benedetti, C. B. Schroeder, C. G. R. Geddes, J. Van Tilborg et al., Tunable laser plasma accelerator based on longitudinal density tailoring, Nat. Phys. 7, 862 (2011).

[54] A. Buck, J. Wenz, J. Xu, K. Khrennikov, K. Schmid, M. Heigoldt, J. M. Mikhailova, M. Geissler, B. Shen, F. Krausz et al., Shock-Front Injector for High-Quality Laser-Plasma Acceleration, Phys. Rev. Lett. 110, 185006 (2013).

[55] B. Beaurepaire, A. Vernier, M. Bocoum, F. Böhle, A. Jullien, J. P. Rousseau, T. Lefrou, D. Douillet, G. Iaquaniello, R. Lopez-Martens et al., Effect of the Laser Wave Front in a Laser-Plasma Accelerator, Phys. Rev. X 5, 031012 (2015).

[56] M. Chen, Z.-M. Sheng, Y.-Y. Ma, and J. Zhang, Electron injection and trapping in a laser wakefield by field ionization to high-charge states of gases, J. Appl. Phys. 99, 056109 (2006).

[57] A. Pak, K. A. Marsh, S. F. Martins, W. Lu, W. B. Mori, and C. Joshi, Injection and Trapping of Tunnel-Ionized Electrons into Laser-Produced Wakes, Phys. Rev. Lett. 104, 025003 (2010).

[58] M. Chen, E. Esarey, C. B. Schroeder, C. G. R. Geddes, and W. P. Leemans, Theory of ionization-induced trapping in laser-plasma accelerators, Phys. Plasmas 19, 033101 (2012).

[59] T. D. Arber, K. Bennett, C. S. Brady, A. LawrenceDouglas, M. G. Ramsay, N. J. Sircombe, P. Gillies, R. G. Evans, H. Schmitz, A. R. Bell et al., Contemporary particle-in-cell approach to laser-plasma modelling, Plasma Phys. Controlled Fusion 57, 113001 (2015).

[60] A. Nause, E. Dyunin, R. Ianconescu, and A. Gover, Exact theory of optical transition radiation in the far and near zones, J. Opt. Soc. Am. B 31, 2438 (2014).

[61] J. D. Jackson, Classical Electrodynamics (Wiley, New York, 1998).

[62] J. Faure, C. Rechatin, A. Norlin, A. Lifschitz, Y. Glinec, and V. Malka, Controlled injection and acceleration of electrons in plasma wakefields by colliding laser pulses, Nature (London) 444, 737 (2006).

[63] H. Kotaki, I. Daito, M. Kando, Y. Hayashi, K. Kawase, T. Kameshima, Y. Fukuda, T. Homma, J. Ma, L-M. Chen et al., Electron Optical Injection with Head-on and Countercrossing Colliding Laser Pulses, Phys. Rev. Lett. 103, 194803 (2009).

[64] G. Golovin, W. Yan, J. Luo, C. Fruhling, D. Haden, B. Zhao, C. Liu, M. Chen, S. Chen, P. Zhang et al., Electron Trapping from Interactions between Laser-Driven Relativistic Plasma Waves, Phys. Rev. Lett. 121, 104801 (2018). 